\title{
Impact of Mobility on MANETs Routing Protocols Using Group Mobility Model
}

\author{
Subodh Kumar ${ }^{a, *}$, G.S. Agrawal ${ }^{\mathrm{a}}$, Sudhir Kumar Sharma ${ }^{\mathrm{b}}$ \\ ${ }^{a}$ Department of Computer Applications, IET, Mangalayatan University, Beswan, Aligarh-U.P. (India) \\ ${ }^{b}$ School of Engineering \& Technology, Jaipur National University, Jaipur-Raj (India)
}

\begin{abstract}
The MANET routing protocols should be tested under realistic conditions of the network including various parameters such as network size, representative data traffic models, realistic movements of the mobile users, etc. Many factors impinge the performance evaluation of MANETs routing protocols. Mobile nodes are communicated with each other with the help of routing protocols. Unpredictable movement of a mobile node affect the routing information which directly interrupt the subsist communication. A mobility model is used to depict the realistic movements of mobile nodes in the designed scenario. In this study the group mobility model has been used to deploy the mobility effect in the scenario. The goal of this paper is to investigate the impact of group mobility on performance of routing protocols under group mobility model using QualNet simulator. In the paper it is illustrate that how the performance results of an ad hoc network protocol drastically change with the increasing node density. The various scenarios investigated with varying density of nodes in groups. Performance analysis is carried out on the basis of performance metrics under group mobility model. The outcome of this work shows that mobility has a detrimental impact on the performance of routing protocols. From the simulation results, it is shown that the DSR protocol clearly outperform all other routing protocols with increasing node density under group mobility model.
\end{abstract}

Index Terms: MANET, Routing Protocols, Mobility, Group Mobility Model.

(C) 2017 Published by MECS Publisher. Selection and/or peer review under responsibility of the Research Association of Modern Education and Computer Science

\section{Introduction}

Ad-hoc network is an infrastructure less network in which collection of mobile nodes forming a temporary network without any centralized control or administration. MANET does not require a pre-existing architecture for communication as all communication occurs through a wireless medium. The interest in ad-hoc network is

\footnotetext{
* Corresponding author.

E-mail address: subodhsharma.86@gmail.com
} 
greatly peaked up with the current technology and the increasing popularity of notebook computers, tablets and I-pods. With the use of latest technology we can form small ad-hoc networks on campuses, during conferences, and even in our own home. However, the main importance of ad-hoc networks can be realized in rescue missions and in situations located in rough or underdeveloped territories. The topology in MANET is not fixed; it gets changed dynamically because of the mobility characteristic of nodes. Sometimes there is a need to realize movement of nodes in form of groups and for the same group mobility model is used. The applications of group mobility may are include military operations, searching and rescue in disaster recovery, visiting an exhibition hall, and firefighters operating in a building [1].

MANETs routing protocols, as an important research topic in MANETs, have gained a lot of interest among the research community. All nodes of MANETs work as router and take part in discovery and maintenance of routes. The availability of routers at an instant can increase or decrease due to mobility hence availability of paths can vary in an Ad-hoc network [8]. A routing protocol is most commonly used for exchanging the information in form of data packets from one node to another.

Mobility has the significant impact on the performance of network routing protocols. The most previous research studies have focused on the individual's mobility behaviors.

However, there is very few studies conducted which describe the group mobility behavior in performance evaluation a routing protocol.

The various routing protocols have been developed to manage the routing in MANET. In general routing protocols are broadly classified into three types such as Proactive, Reactive and Hybrid protocols [2, 3].

(i) Proactive Protocols: These types of protocols are table driven protocols in which, the routes are consistent and up-to-date the routing information to all nodes. Packets are transferred over the available route specified in the routing table. Proactive protocols have lower latency because all available routes are maintained at all times for all available nodes. Some of the proactive routing protocols are DSDV, OLSR, WRP, FSR, and CGSR.

(ii) Reactive Protocols: These types of protocols are also known as On Demand Routing Protocols where routes are not maintained before transferring the packets for routing. When a source wants to send information to a destination, it invokes the route discovery mechanisms to find the path to the destination. This route discovery is done by flooding mechanism, in which a source node just broadcasts the packet to all of its neighboring and intermediate nodes and forward that packet to their neighbors until it reaches to the destination. Reactive techniques have smaller routing overheads because of no prior routing information requirement, but higher latency. The Reactive Protocols are much suited and perform better for Ad hoc networks. Some of the Reactive Routing Protocols are: DSR, AODV, LAR, and ABR.

(iii) Hybrid Protocols: Hybrid protocols comprises the features of both reactive and proactive routing protocols and take the advantages of both protocols which results in quick routes discovery in the routing zone. Some of the Hybrid Routing Protocols are: ZRP, TORA, and HSLS.

The routing protocols perform better in case of fixed and stationary environment but in case of mobility scenario the performance of routing protocol may distress drastically because of the dynamic change in topology. The dynamic and unpredictable changes in topology are the main cause of route failure which destroys the communication deficiently. In this paper we have presented performance evaluation of five proactive routing protocols (Bellman ford, Fisheye, LANMAR, RIP, and STAR), three reactive routing protocols(AODV, DSR, and DYMO) and hybrid (ZRP) routing protocol with varying node density (20, 60, 100, 140, 180 and 200) under the group mobility using Qualnet simulator. We have compared the performance of these routing protocols using three performance metrics (Average Throughput, Average Jitter, and Average End-to-End Delay). The paper is divided in following sections: Section II covers the brief introduction to mobility, Data traffic model and performance metrics. In section III the related work is summarized. In section 
IV the simulation scenario and related parameters is explained. Section $\mathrm{V}$ describes the analysis and results discussion part and. Section VI provides the conclusion followed by references.

\section{Mobility, Data Traffic Model and Performance Metrics}

\section{(i) Group Mobility Model}

In order to evaluate the performance of a routing protocol, it is necessary to use mobility a model. The mobility model is designed to describe the movement pattern of mobile users, and to predict how their location, velocity and acceleration change over time [8]. To generate the mobility effect in simulation scenarios various mobility models has been proposed [4-7]. In this work the group mobility model have been used to generate the group mobility pattern. In this mobility model, group of nodes move together. The movement of a group follows the Random Waypoint model and if any node moves within the group area also follow the Random Waypoint model [9]. The communication between the different groups is shown below in fig.- 1 .

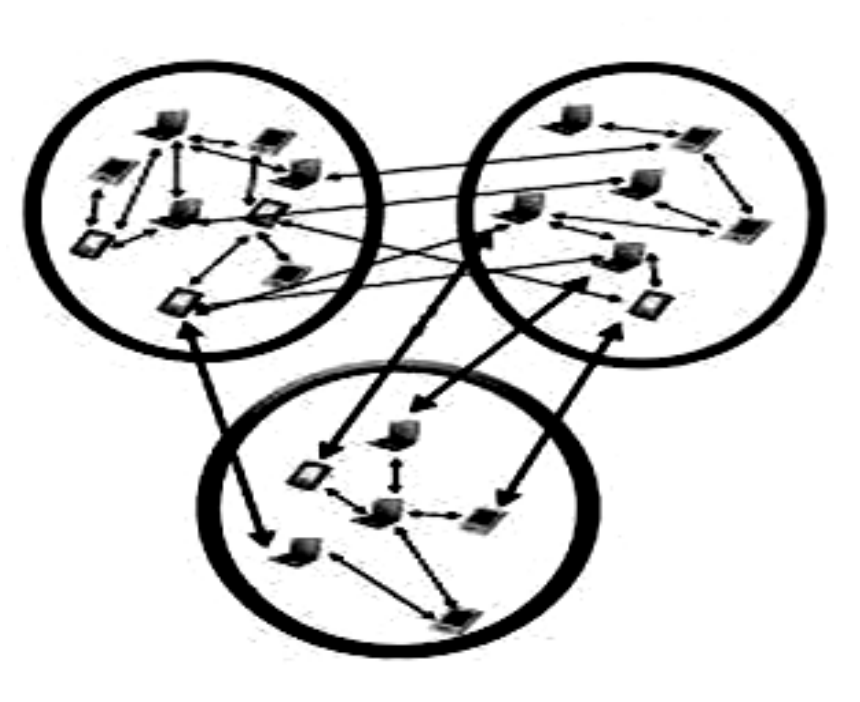

Fig.1. Communication between Groups

\section{(ii). Data Traffic Model}

The CBR is used for connections that transport traffic at a constant bit rate in real time between communication pair (source and destination). CBR is tailored for any type of data for which the end-systems require predictable response time and a static amount of bandwidth continuously available for the life-time of the connection [10]. In simulation design the 4 CBR connections have been created.

\section{(iii). Performance Metrics}

Different performance metrics are used to investigate the impact of group mobility on the performance of routing protocols. The performance metrics are generally representing the diverse characteristics of the overall network performance. The following performance metrics are used to evaluate the performance of said routing protocols: 
a. Average End-to-End Delay Average End-to-End Delay performance metrics is used to measure the time taken by a packet to travel across a network from a source node to the destination node.

b. Average Jitter Average jitter is a performance characteristics used to measure deviation from true periodicity eventually of inactivity in packet across network.

Jitter in IP networks is the variation in the latency on a packet flow between two systems, when some packets take longer to travel from one system to the other. Jitter results from network congestion, timing drift and route changes. The most effective condition of jitter is shown in fig.-2.

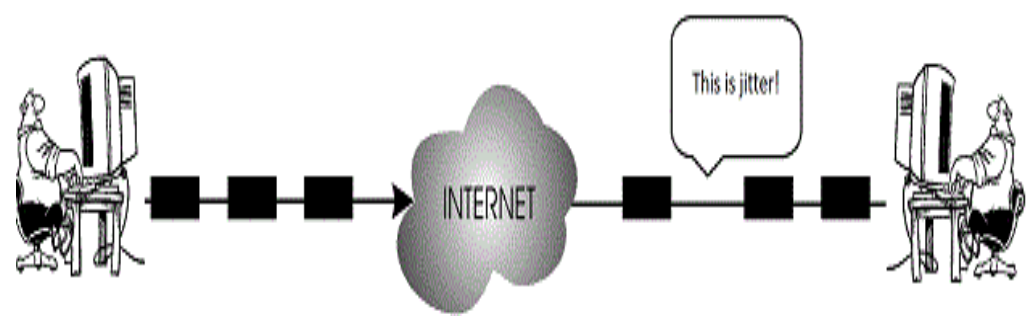

Fig.2. Jitter

c. Throughput Throughput is measured in terms of successful delivery of data packet within the threshold time.

\section{Related Work}

This section presents a brief review of literature on performance evaluation of routing protocols under group mobility model in MANETs. In MANETs movement of a node from one place to another characterize mobility, thus mobility is directly responsible for the link failure. Mobility is an important factor for MANETs and it plays a vital role in routing protocols performance evaluation [8].

In MANET, routing protocols are typically categorized into two classes, table-driven routing protocols and on-demand routing protocols $[11,12]$. However, the combination of both the approaches represents the hybrid strategy. N.Aschenbruck, et.al. [13] have discussed various mobility models for MANETs. These are required to model mobile nodes in various scenarios in simulation studies and show their varying speeds and connectivity. Prabhakaran and Sankar [14] have studied the impact of entity and group mobility models on the performance of MANET with one reactive routing protocol i.e. AODV. The MANET performance is affected by the mobility of the nodes; therefore, the choice is to be made wisely regarding the usability of any mobility model. In Bai, Fan, et.al, discussed that mobility model plays a vital role in protocol comparison. The most commonly used mobility model is Random Waypoint model [15] in Manet's simulation studies. The performance evaluation of two routing protocols namely Optimized Link State Routing-Institut national de recherché en informatique et an automatique (OLSR-INRIA) and Dynamic Opportunistic Routing (DOR) have been evaluated by Kulshrestha S. and Trivedi A. [16]. Through the evaluation study they have found that the network density plays an important role in the performance evaluation of routing protocols. Goswami S., Agrawal C., and Jain A.[17], have evaluated the performance of AODV and LAR. Through the simulation study they have found that the LAR protocol reduces the energy consumption. The performance of DYMO and AODV protocol using QualNet simulator have been analyzed by Hakak et al.[18]. They have performed the simulation study using 100 nodes with random waypoint mobility model with the max speed $30 \mathrm{mps}$. The results of the study indicated that to keep average jitter at optimum level is to give priority to packet size followed by routing protocol as both of these factors has significant impact on average jitter. A highly dense environment is simulated for varying nodes using OLSR, AODV and DSDV routing protocols by Siakoulis et al.[19]. In the study they have revealed that the performance of various routing protocols varies for different 
scenarios. In particular, the AODV indicates quite satisfactory performance in terms of throughput and end-toend delay. The OLSR performs well in terms of throughput while on other side it increases the routing overhead in the denser situations. The DSDV has a better throughput when the number of connection in the network increases. The performance comparison of DSR, AODV, and DSDV routing protocol for different metrics using simulation method have been conducted by Mehmood et al.[20]. They have indicated in the results that the AODV perform well than that of other routing protocols. Sharma S. et al. have conducted a study on impact of mobility models on MANETs routing protocols. In their study they have summarized that the protocol performance may change drastically across mobility models and performance rankings of protocols may vary with the mobility models.

\section{The simulation scenario and related parameters}

\section{(i) Simulation Scenario}

In this paper, an attempt has been made to study and investigate the impact of group mobility with the help of QualNet simulator. The various scenarios designed to evaluate the performance of nine routing protocols: Bellman ford, Fisheye, LANMAR, RIP, STAR, AODV, DSR, DYMO, and ZRP with CBR traffic patterns and varying node density using Group mobility model.

The throughput, average end-to-end delay and average jitter performance metrics have been used to evaluate the performance of routing protocols. The simulation setup is executed for 120 seconds. In table- 1 , the simulation parameters are presented in detail. The MANET consists of node density 20,60,100, 140, 180 and 200 which are distributed in four equal groups in each case of node density in terrain size of $1500 \mathrm{mx} 1500 \mathrm{~m}$. The data packet size is of 2048 bytes used.

Table 1. Simulation parameters

\begin{tabular}{|l|l|}
\hline Simulation parameters & Value \\
\hline Terrain Size & $1500 * 1500$ \\
\hline Node Placement Strategy & Group \\
\hline Traffic source & CBR(4 Connections) \\
\hline Network size (Number of nodes) & $20,60,100,140,180,2000$ \\
\hline Protocols & $\begin{array}{l}\text { Bellman ford, Fisheye, LANMAR, RIP, STAR, AODV, DSR, } \\
\text { DYMO, and ZRP }\end{array}$ \\
\hline Mobility Model & Group Mobility Model \\
\hline Item size & 5000 bytes \\
\hline MAC protocol & 802.11 \\
\hline Radio Type & $802.11 \mathrm{~b}$ \\
\hline Group Mobility Pause & 1000 mille second \\
\hline Group Mobility Speed(Min, Max $)$ & $(5 \mathrm{mps}, 10 \mathrm{mps})$ \\
\hline Group Mobility Internal Speed(Min, Max) & $(5 \mathrm{mps}, 10 \mathrm{mps})$ \\
\hline Antena Model & Omni directional \\
\hline Simulation time & 120 seconds \\
\hline
\end{tabular}


The following fig.-3 representing the group of 140 node density having the four groups (group0, group1, group2 and group3) of 35 nodes each. The type of various scenarios has been designed for different-different node density $(20,60,100,180,200)$ and simulation conducted. Every scenario of distinguish density is simulated for 120 seconds and every environment is equally divided in four groups of equal number of node density for every density of node.

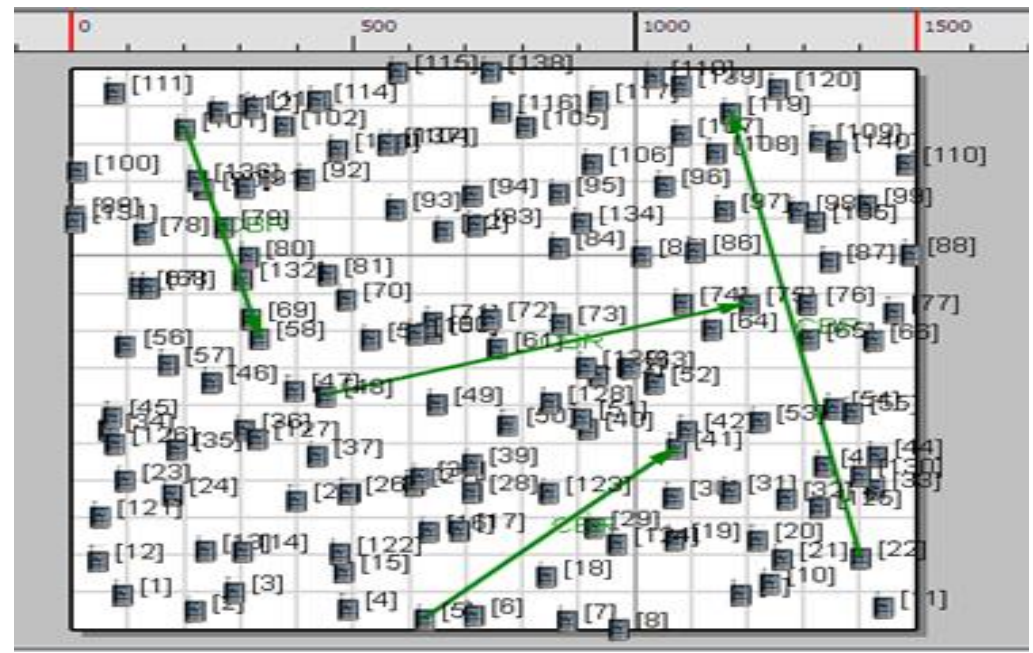

Fig.3. Scenario of 140 nodes having 4 groups of equal node density

In this study the CBR data traffic model is applied on 4 pair $[(101,58),(48,75),(5,41),(22,119)]$ of source and destination nodes. The fig.-4 indicating the applied CBR applications in the scenario between the source and destination. The CBR connections are used to transport the traffic at a constant bit rate in real time between communication pair (source and destination). In general, CBR is tailored for any type of data for which the end-systems require predictable response time and a static amount of bandwidth continuously available for the life-time of the connection.

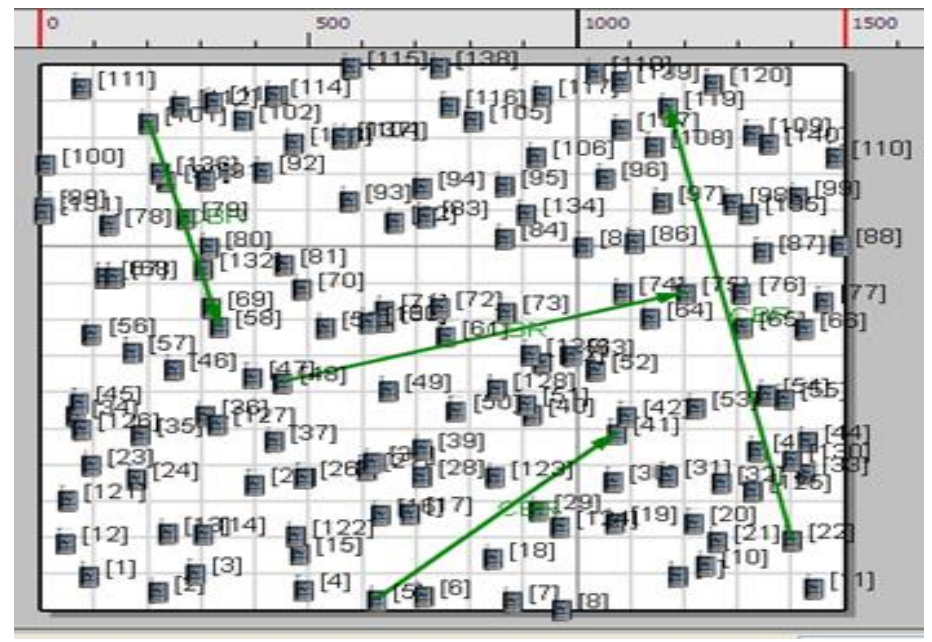

Fig.4. CBR Application 


\section{(ii) Animation View of the Simulation Scenario}

The fig. -5 shows the animation view of the run time simulation of the group mobility. Fig. 5 indicates the real time communication process with in the designed environment between the different source and destination nodes. Each movement of a node is traced and indicated in the following figure in green color. Any used in QualNet can see the node movement in the designed scenario through the animation view. The following fig.-5 is only the screen shot of the animation view of a particular moment of time.

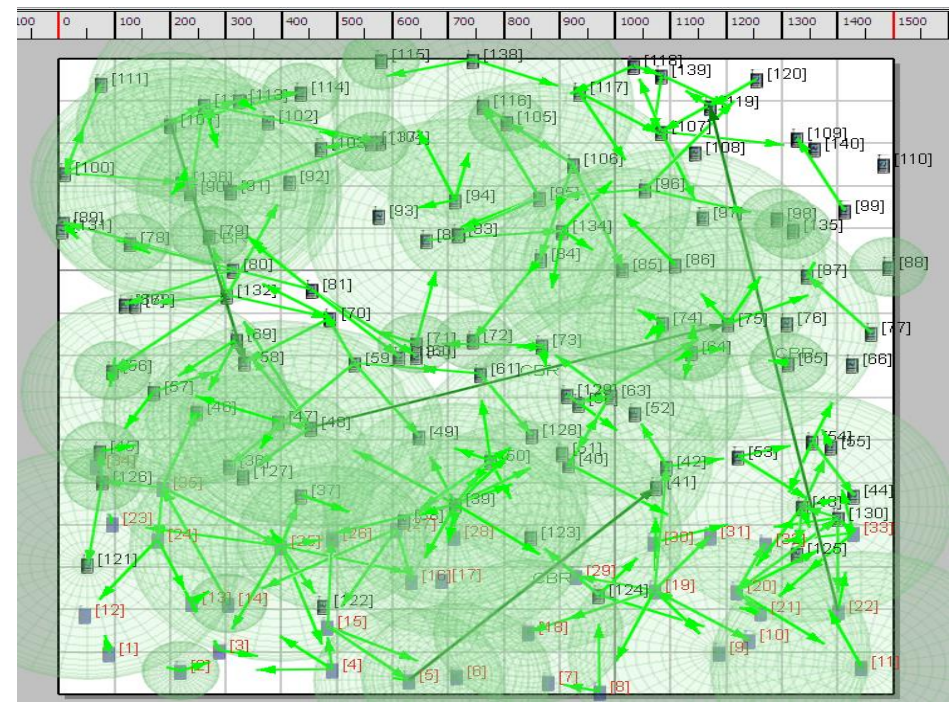

Fig.5. Animation View of Simulation

\section{The Results and Discussion}

In this section, the performance comparison of Bellman ford, Fisheye, LANMAR, RIP, STAR, AODV, DSR, DYMO, and ZRP routing protocols under group mobility model with varying node density is presented. The performance of above routing protocols has been analyzed using performance metrics. The simulation results are shown in the form of graphs that represents (i) Throughput (Kb/s) (ii) Average End- to- End Delay (s) and (iii) Average Jitter(s)

\section{(i) Average Throughput}

The performance comparison of Bellman ford, Fisheye, LANMAR, RIP, STAR, AODV, DSR, DYMO, and ZRP routing protocols under group mobility model with varying node density is conducted. The results are shown in fig.-6. The outcome of throughput of different routing protocols is divided by 1000 and rounded up to two decimal places in order to minimize the output values of throughput for the ease of analysis. From the fig.-6, it clearly indicates that the DSR outperform to all other routing protocols in case of throughput with increasing node density and ZRP routing protocol gives nastiest results in all cases. The performance of DSR is quite satisfactory with increasing node density however, DSR indicate downfall in term of throughput especially at 100 node density. From the CBR server results it is observed that it happens because of the significant downfall in packet delivery ratio and transmission delay at that juncture. On other hand, AODV shows quite good results for the high node density scenarios particularly for more than 100 nodes. However, 
Fisheye protocol shows good performance in case of low node density, and for more than 100 nodes there is tremendous downfall in term of their performance.

The LANMAR and STAR routing protocols show moderated downfall. On other hand, DYMO and RIP indicate increasing performance for high node density particularly from 140 nodes. The Bellman Ford routing protocol indicate good results in case of high node density than the low node density. Form the fig. it is very clearly indicating that the 100 node density is a very critical point where the performance of all most routing protocols goes up while RIP and FISHEYE protocols indicate their performance in term of downfall to 60 node density. The moderated improvement is observed in RIP protocol from 100 nodes onwards however the FISHEY protocol clearly shows downfall with the increasing node density from 60 nodes onwards.

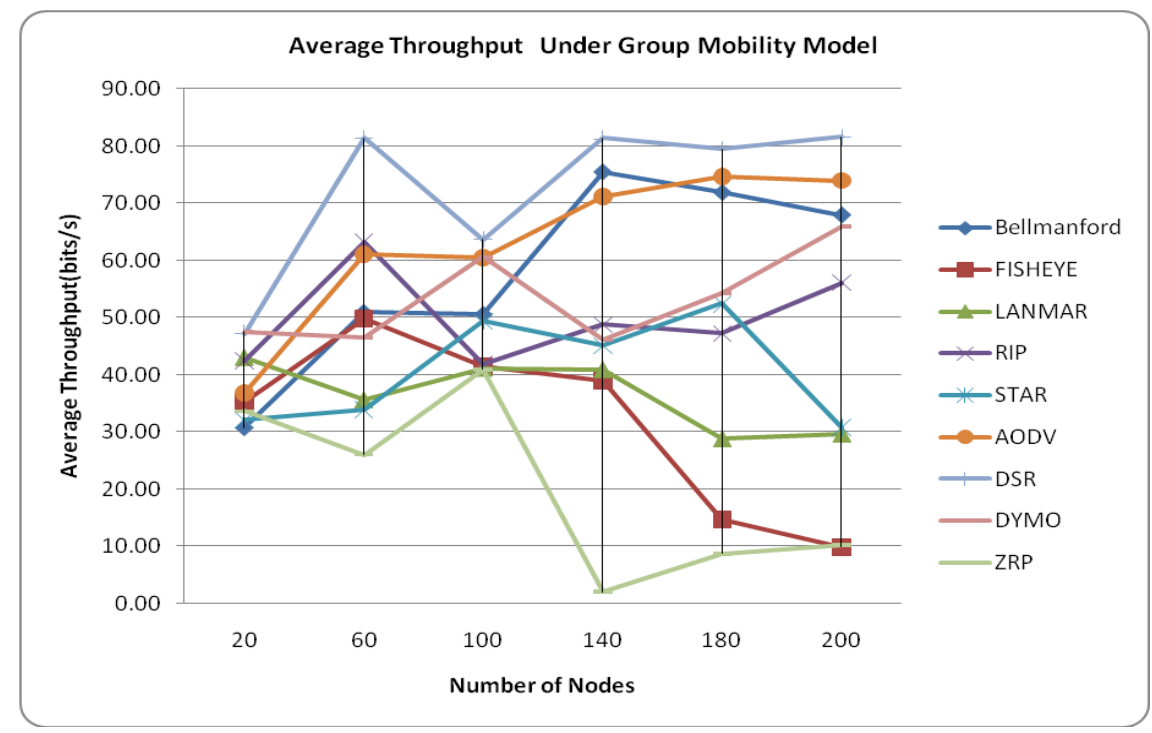

Fig.6. Throughput Vs Node

\section{(ii) Average End-to-End Delay}

The performance comparison of Bellman ford, Fisheye, LANMAR, RIP, STAR, AODV, DSR, DYMO, and ZRP routing protocols under group mobility model with varying node density is conducted. The results of average end-to-end delay are shown in fig.-7. From the fig.-7 it is clearly indicating that the STAR routing protocol clearly outperform all other routing protocols incase of average end-to-end delay. The Bellman ford and LANMAR perform poor for less than 100 node density and DSR particularly perform poorly for 20 nodes. It happened because of delay in route discovery and packet arrival at this point. However, the Fisheye routing protocol gives worst results in high node density for the delay particularly from 100 node density as it shows extensive increase in delay. It happened because of the high delay in packet delivery particularly it starts from 100 node density onwards. On other hand, the ZRP routing protocol shows worst performance in all cases. In general, the variation in delay arises due to various reasons such as delay in packet delivery, route discovery, and route recovery and route formation. 


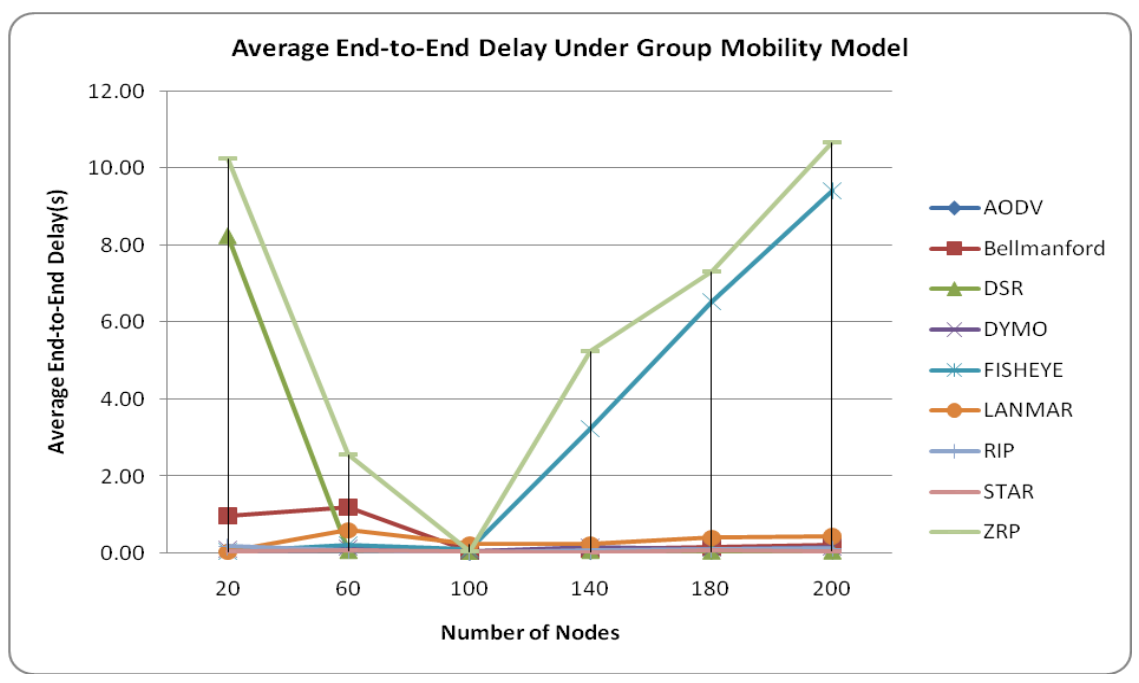

Fig.7. Average End-to-End Delay Vs Node

\section{(iii) Average Jitter}

The performance comparison of Bellman ford, Fisheye, LANMAR, RIP, STAR, AODV, DSR, DYMO, and ZRP routing protocols under group mobility model with varying node density is conducted. The results of average jitter are shown in fig.-8. From the fig.-8 it is clearly indicated that the STAR routing protocol clearly outstretch all other routing protocol incase of increasing node density for the average jitter. ZRP routing protocol shows inferior performance in almost cases of increasing node density up to 160 nodes and for the more than 160 nodes FISHEYE gives worst performance in comparison of ZRP routing protocol. However, FISHEYE perform well in low node density scenario particularly up to 160 nodes. On other hand, LANMAR routing protocol indicates moderated less performance in comparison to all other routing protocols apart from ZRP and FISHEYE with increasing node density. However, the DYMO, RIP and AODV show mediocre performance in term of average jitter. The variations of average jitter are observed because of the deviation in packet inter-arrival time to their destination and high network congestion.

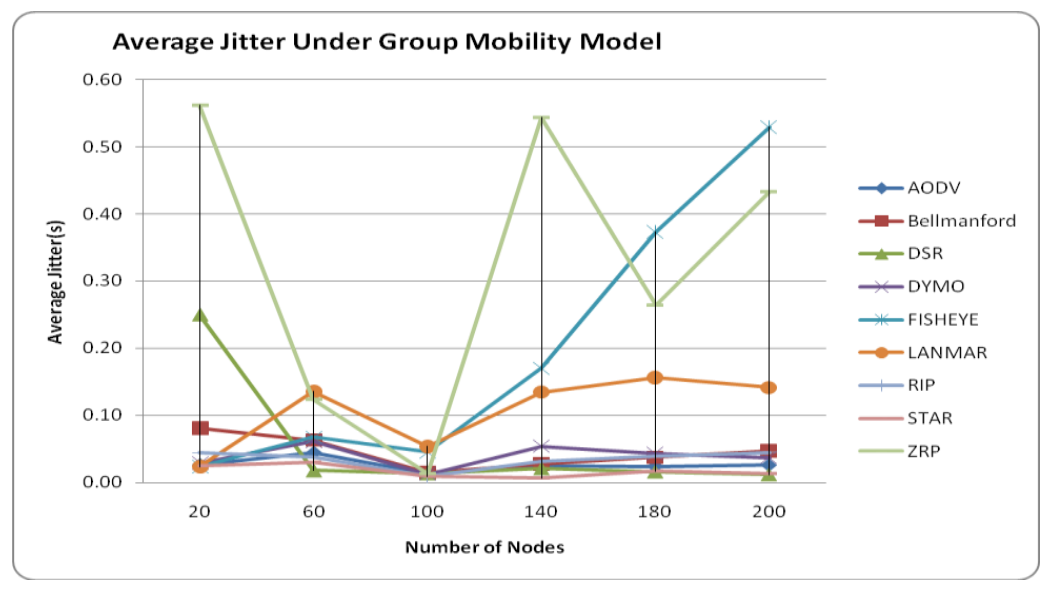

Fig.8. Average Jitter 


\section{Conclusions}

In this paper performance evaluation of five proactive routing protocols (Bellman ford, Fisheye, LANMAR, RIP, and STAR), three reactive routing protocols (AODV, DSR, and DYMO) and hybrid (ZRP) routing protocol with varying node density $(20,60,100,140,180$ and 200) using QualNet simulator is presented. The three performance metrics Average Throughput, Average Jitter, and Average End-to-End Delay under group mobility model is used for performance evaluation of routing protocols. Till now many routing protocols have been used in MANETs, each having unique features.

From above analysis part it is clear that the group mobility has significant impact on the performance of all type of routing protocols. Simulation results show that group density in groups and their mobility have a significant impact on the network performance which has not been revealed before. However, choosing an efficient routing protocol is a critical task for study the operations and performance of MANETs.

It is clearly indicating that reactive routing protocols AODV, DSR and DYMO are best suited in large dense scenarios for group mobility. On other hand, proactive routing protocols Bellman ford, Fisheye, LANMAR, RIP, and STAR are not show good performance as with the increasing node density the routing overhead increase simultaneously i.e. thousands of routing tables to be maintained by the proactive routing protocols in large MANET that actually degrades the efficiency of routing protocols. So, for highly dense and large groups MANET routing protocols gives quite satisfactory results than that of proactive routing protocols. However, the hybrid routing protocol ZRP connote the nastiest performance in case of all performance metrics. Hence, the hybrid routing protocols are not suited for MANET with large groups type of scenarios.

\section{References}

[1] Yan Zhan et.al. "Performance Evaluation of Routing Protocols on the dereference Region Group Mobility Model for MANET”, Wireless Sensor Network, 3, pp. 92-105, 2011.

[2] Laura Marie Feeney. A taxonomy for routing protocols in mobile ad hoc networks. Technical report, Swedish Institute of Computer Science, Sweden, 1999.

[3] http://www.telenor.com/rd/pub/rep03/R_41_2003.pdf

[4] Tracy Camp et.al., "A Survey of Mobility Models for Ad Hoc Network Research" , Wireless Communication \& Mobile Computing (WCMC): Special issue on Mobile Ad Hoc Networking: Research, Trends and Applications, vol. 2, no. 5, pp. 483-502, 2002.

[5] Nicholas Cooper and Natarajan Meghanathan,"Impact of Mobility Models in Multi-Path Routing in Mobile Ad Hoc Networks", International Journal Of Computer Networks \& Communications (IJCNC), Vol. 2, No.1, 2010.

[6] Michael Feeley et.al, "Realistic Mobility for Mobile Ad Hoc Network Simulation", I. Nikolaidis et al. (Eds.): ADHOC-NOW 2004, LNCS 3158, pp. 324-329, 2004.

[7] Amit Jardos et.al., “Towards Realistic Mobility Models For Mobile Ad hoc Networks",MobiCom'03, September 14-19, San Diego, California, USA, 2003.

[8] S. Kumar et.al. "Impact of Mobility Models on MANETs Routing Protocols", Inroads, Vol. 3, Print ISSN: 2277-4904. Online ISSN: 2277-4912, pp. 142-147, 2014.

[9] QualNet-5.1 Users Guide.pdf

[10] Ferguson P., Huston G., "Quality of Service: Delivering QoS on the Internet and in Corporate Networks", John Wiley \& Sons, Inc., ISBN 0-471-24358-2, 1998.

[11] M. Bouhorma, H. Bentaouit and A. Boudhir, "Performance Comparison of Ad-Hoc Routing Protocols AODV and DSR,' International Conference on Multimedia Computing and Systems (ICMCS'09), Ouarzazate, 2-4 April, pp. 511-514, 2009.

[12] E. M. Royer and T. Chai-Keong, "A Review of Current Routing Protocols for Ad Hoc Mobile Wireless Networks,” IEEE Personal Communications, Vol. 6, No. 2, pp. 46-55, 1999. 
[13] N.Aschenbruck et.al. "A Surveyon mobility models for Performance analysis in Tactical Mobile networks," Journal of Telecommunication and Information Technology, Vol.2 pp.54-61,2008.

[14] P Prabhakaran and R Sankar, "Impact of realistic mobility models on wireless networks performance", In Proceedings of the IEEE International Conference on Wireless and Mobile Computing, Networking and Communications, Montreal, QC, Canada, 19-21 June; pp. 329-334, 2006.

[15] Bai Fan et.al, "A Survey of Mobility Models in Wireless Adhoc Networks", Chapter 1 in Wireless AdHoc Networks. Kluwer Academic, 2006.

[16] Kulshrestha S. and Trivedi A., "An Approach Towards Dynamic Opportunistic Routing in Wireless Mesh Networks", I.J. Wireless and Microware Technologies,2, pp. 26-32, 2012.

[17] Goswami S., Agrawal C., Jain A., "Location based Energy Efficient Scheme for Maximizing Routing Capability of AODV Protocol in MANET", I.J. Wireless and Microware Technologies,3, pp. 33-44, 2015.

[18] Hakak, S., Latif, S. A., Anwar, F. and Alam, M.K., "Impact of Key Factors on Average Jitter in MANET", First International Conference on Systems Informatics, Modeling and Simulation. Computer Society, IEEE, pp. 179-183, 2014.

[19] Siakoulis, Y., Galiotos, P. Dagiuklas, T. and Kotsopoulos, S., "The impact of simulation duration on the performance of the OLSR, AODV and DSDV Protocols, in a heavy-loaded Ad-hoc wireless mobile environment", First international conference on system informatics and modeling, IEEE, pp. 160-167, 2014.

[20] Mehmood, Z., Iqbal, M. and Wang, X., "Comprehensive experimental performance analysis of DSR, AODV and DSDV routing protocol for different metrics values with predefined constraints", IJITCS, 6(7), pp. 24-31, 2014.

[21] Kumar S., Agrawal G.S., and Sharma S.K., Impact of Mobility Models on MANETs Routing Protocols", INROADS, 3(1), pp. 142-147, 2014.

\section{Authors' Profiles}

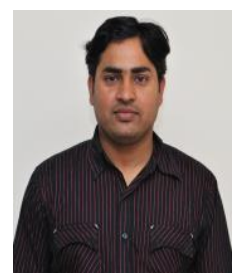

Dr. Subodh Kumar Sharma is Head in Department of Computer Applications, Institute of Engineering and Technology, Mangalayatan University, Aligarh. He has obtained his Ph. D. from Mangalayatan University, Aligarh and the Master degree in computer application from Dr. A.P.J. Abdul Kalam Technical University, Uttar Pradesh, Lucknow (formerly known as Uttrapradesh Technical University, Lucknow). He has been meticulously carried out research activities since last 8 years in the field of Mobilie Ad-hoc Network, Software Engineering and Database. He has taught various courses at graduate as well as at postgraduate level and supervised number of projects and dissertations of BCA \& MCA students. Presently he is supervising four Ph. D scholars in the field of Big Data and Mobile Ad-hoc Network. He has published many research papers in International Journals of repute, and has been part of many National and International conferences.

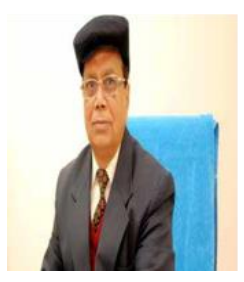

of Fluid Flows.
Prof. (Dr.) G.S. Agrawal is a distinguished Academic personality with Ph. D. from Indian Institute of Technology, Kanpur in the year 1970. He has served in Indian Institute of Technology Roorkee for 40 years in the Department of Mathematics. At present he is serving as Controller of Examinations and Director, Institute of Computer Applications in Mangalayatan University, Aligarh. He has published more than 50 papers in International Journals of repute, and he supervised number of $\mathrm{Ph}$. D scholars and research projects. His research areas include Mobile Ad-hoc Networks, Hydrodynamic and MHD Stability theory 


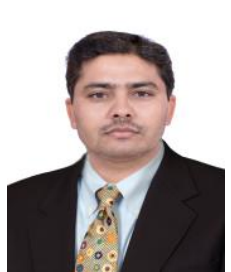

Prof. (Dr.) Sudhir Kumar Sharma is Professor \& Head at the Department of Electronics \& Communication Engineering, School of Engineering and Technology, Jaipur National University, Jaipur, Rajasthan, India. Professor Sharma received his Ph.D. in Electronics from Delhi University in 2000. Professor Sharma has an extensive teaching experience of 17 years. He has been keenly carrying out research activities since last 19 years prominently in the field of Optical Communication. He has taught various engineering courses at graduate as well as at post-graduate level in India and Overseas. His area of research includes Optical Communication, Nonlinear Optics and Optical Sensors. He has authored and coauthored over 60 National and International publications along with one book in Opto-Electronics titled "Introduction to Opto-Electronics and Optical-Communication", Prentice Hall, Malaysia. He has also served on the editorial board of six international journals. He has been part of many National and International conferences worldwide. Professor Sharma continues to serve on many academic, professional and governmental advisory committees. He has organized numerous National and International Workshop, Seminar and Conferences. He has visited Spain, Holland, Malaysia, Ethiopia, Sudan and Syria for academic and research related assignments. He has awarded Senior Research Fellowship by CSIR, New Delhi in 1997. He has also been honored with the prestigious Shiksha Rattan Puruskar by International Friendship Society, New Delhi for academic works in Feb. 2013.

How to cite this paper: Subodh Kumar, G.S. Agrawal, Sudhir Kumar Sharma,"Impact of Mobility on MANETs Routing Protocols Using Group Mobility Model", International Journal of Wireless and Microwave Technologies(IJWMT), Vol.7, No.2, pp.1-12, 2017.DOI: 10.5815/ijwmt.2017.02.01 